\title{
Autism and Social Media: The Case of Brazil
}

\author{
Débora Antunes*, Alexander Dhoest** \\ * University of Antwerp, Belgium - Faculty of Social Sciences \\ ** University of Antwerp, Belgium - Faculty of Social Sciences
}

Abstract

\begin{abstract}
This paper presents an overview of how social media is used by Brazilian autism communities, aiming at including the perspectives of the Global South into discussions surrounding the topic, which are mostly focusing on English speaking groups. The results are based on a digital ethnography conducted in three Facebook groups about autism. We observed that groups composed by parents looking for information and social support are predominant in Brazilian social media. Autistic people also engage in information gathering and the exchange of personal experiences, mainly the ones with Asperger Syndrome. Additionally, we saw that Facebook offers enough affordances to allow the participation of people with different levels of digital literacy. However, there is still a lack of representation from autistic people who have more challenges, such as non-verbal individuals, which may be a consequence of the digital divide, not only in terms of economical means, but also education and inclusion.
\end{abstract}

Keywords: autism; social media; Brazil

\section{Introduction}

The Internet is an empowering tool not only for autistic people but also for their parents. For the former, digital media provides a friendly environment that may compensate for the difficulties in communication and social interaction, common challenges of autism, and also possibilities to focus on special interests, the so called hyper focus. Meanwhile, for the autism community, which is formed by autistic people and their parents, the Internet enables the formation of groups where they can exchange information, provide social support to each other, and compensate the exclusion from social life that they often face when a diagnosis is received in the family. A third group formed by extended family members, professionals, and people interested in autism can also benefit from online communication by joining groups about autism to either share knowledge or learn more about autism.

Despite the evident importance of digital media for the communities that surround autism, there is a lack of research on countries of the Global South (Meekosha, 2011), as most scholars concentrate their efforts on English speaking communities. The importance of addressing countries in the Global South is evidenced when we understand disability - and thus autism - from a social perspective, which sees disability as socially constructed and, consequently, based on its regional context. Social media usage can also vary greatly across different regions, as a social media platform is mostly what people do with it rather than merely what it was designed to be (Miller et al., 2016). Based on these ideas, this article seeks to explore online autism communities on Facebook in Brazil. The discussion presented here is based on a literature review and a digital ethnographic study conducted in Brazilian social media. During the initial stages of our research, we utilised a multi-sited online approach to observe the most common platforms used to talk about autism, but it soon became clear that Facebook was predominant and could also incorporate other platforms. Therefore, after the initial stage the focus was narrowed to Facebook and digital ethnography, which consists of an online participant observation, was used to investigate the online cultural aspects of 
autism in Brazil. In this paper, we present the results of our exploratory research, giving the reader an idea of the different groups and practices surrounding autism in the Brazilian cyberspace.

In order to discuss the presence of autism on Facebook in Brazil, we first provide a theoretical discussion of autism and social media, followed by an overview of the methodology we used to gather the results. Then, in the analytical section, we present the main groups that are active in the conversation about autism in Brazil and analyse how they use social media in relation to autism. Finally, we conclude by highlighting some aspects of the online autism community in Brazil and discussing broader implications of our research. Before we start, it is important to point out that the digital ethnography carried out for this research focused on groups that have autism as their main topic, but autistic people and their supporters also use social media for other purposes. As Lister et al. (2009) point out, the Internet offers the possibility of creating a fragmented identity, in a process that they call bricolage; thus, here we concentrate on understanding one facet of this identity, the one related to autism.

\section{Social Media, Facebook, and Autism}

For this research, we were particularly interested in social media, i.e. platforms featuring the possibility of creating a profile and sharing connections and information in different ways (boyd, 2011). Those platforms afford human interaction and collective articulation, which may reflect and feed back into offline identities and culture (Stucliffe et al., 2011). It is also worth noticing that social media not only offer a place where audiences can interact, but they also shape how interaction can happen. As boyd (2011) mentions, the public is transformed by the potential and properties of social media. Hence, social media shape how participants of this research will communicate among each other, creating new venues and possibilities, bringing both benefits and drawbacks, for representations and articulations around autism. Recuero (2011) says that social media often afford reactive and mutual interactions, the former through the use of buttons (e.g. the 'Like' button on Facebook) and the latter based on dialogue.

The use of Internet by disabled people and their supporters has been documented by scholars in the fields of both Internet Studies and Disability Studies (Shpigelman \& Gill, 2014; Ellis \& Goggin, 2011; Seymour \& Lupton, 2004). Regarding autism specifically, scholars have concluded that autistic people can draw a number of advantages from Computer Mediated Communication (CMC), despite the perceived risks and vulnerabilities they may face (Shane-Simpson et al., 2016; van der Aa et al., 2015; Davidson et al., 2012; Newton et al., 2009). Silberman (2015, p. 257-258) emphasizes that "computer networks held the potential for not just 'augmenting' communication but making it possible, period - minus the stuff that normally made conversation so arduous, such as eye contact, body language, tone, and the necessity of making a good impression." He adds that the practical constraints of communicating online also required many implicit aspects of social interaction to be made explicit, giving people with autism enough social cues to make communication easier, such as the use of hashtags (e.g. \#sarcasm) to explicitate the tone of a message. Moreover, Davidson (2008) explains that online communication empowered autistic people and helped them to validate their identities, not framing the diagnosis according to negative medical terms, while also offering them a more controllable environment in relation to sensory overload (Davidson, 2008; Brownlow \& O'Dell, 2006; Bowker, 2003). 
Scholars have also studied how parents of disabled people use online communication (Mustafa et al., 2015; Carter, 2009). As parents of autists face more profound levels of social exclusion than parents of other disabled people (Vasilopoulou \& Nisbet 2015, Clifford 2011), the Internet offers the possibility to socialise among equals and even create a sense of family, exchanging information and finding social support (Reinke \& Solheim, 2015). In more general terms, research about autism and the Internet has been growing with the popularisation of autism diagnoses world-wide and the high number of reports about the importance of the Internet in relation to autism (Silberman, 2015; Benford \& Standen, 2009; Dekker, 2006).

Online articulations in relation to autism also affect the social construction of the term. Ortega et al. (2013) affirm that as caregivers and autistic people become more informed and aware of biomedical proceedings, they have more power to influence and decide about therapies, further research, and what it means to be autistic. They add that "online communities are not passive products on the Internet, they have an active role in the construction of diagnosis as well as how the experiences can be socially understood" (Ortega et al., 2013, p. 129). Hence, being online is not just a matter of social support or exchanging information, but also one of identity and culture, aspects that we seek to better understand in our study.

With these ideas in mind, the study of people with autism and digital culture is of undeniable importance. Additionally, the importance of studying digital practices according to their socio-cultural location is strongly stressed by Stein-Sparvieri (2012), whose work shows that the use of digital media by people with disability changes according to the regional context: in places where disability laws are stronger, people tend to use digital media to discuss questions related to community, the social construction of disability and identity politics, while in regions where basic rights and accessibility are issues, digital practices focus on limitations and economical aspects. Critical Autism Studies scholars, who deal with the discourses and power relations behind the concept of autism, have also commented upon the differences of autism across countries, O'Dell et al. (2016) pointing out that different socio-cultural contexts change the definition of autism and how people deal with it. Thus, in the analysis, after presenting the methodology, we will discuss what we could observe while carrying a digital ethnography in Brazilian autism communities on Facebook.

\section{Methodology}

As mentioned, we used digital ethnography to observe online autism communities in Brazil. This method transfers ethnographic practices to the digital environment, allowing the observation and analysis of digital cultures. Broadly speaking, ethnography "consists of a researcher spending an extended period of time immersed in a field setting, taking account of the relationships, activities and understandings of those in the setting and participating in those processes" (Hine, 2000, p. 4-5).

We divided our digital ethnographic study in eight steps: (1) exploratory multi-sited observation, (2) creation of sensitising concepts based on the preliminary observations and readings, (3) selection of participants and obtaining consent, (4) entrance and initial participant observation, (5) selection of the categories of analysis, (6) further participant observation conjoined with elicitation techniques for data 
collection, (7) analysis of data and elaboration of arguments supported by theoretical background, (8) structuring a final narrative of arguments. This paper presents the results obtained during the four initial stages of our research, which allowed us to understand the Brazilian online scene of autism. As studies regarding this subject are scarce across the Global South, we found it necessary to build this narrative in order to proceed with the data analysis and also to give the reader an idea of what we found when going online. What we did in each stage that led to the discussion in this paper is presented in detail below.

Before and during all the stages that led to this paper, we did an academic literature review related to social media and autism. Then, in a first research step, we effectuated an exploratory observation of communities, blogs, profiles and other forms of online communication used by the autism community. Ethnographic studies are usually based on small samples and in depth analysis rather than in shallow analysis of a large sample. However, samples cannot be collected without observation, as it is important to understand the general environment in which samples can be found. As the intention of this research is to observe the digital culture of autism, a multi-sited approach was used for the initial selection, not limited to a single site or platform since "the natural performance of the users as actors in several sites and through several applications does not allow for such a constructed limitation of fieldwork" (BeneitoMontagut, 2011, p. 719). Boellstorff et al. (2012) concur, stating that "multi-sited ethnography may thus be useful for capturing a holistic picture of the life of a community or activity, and the scope of the fieldsite may itself be emergent" (p. 60). Thus, this initial stage was fundamental to give us an idea of the autism community before selecting and focusing on a specific sample.

A second research step implied the creation of sensitising concepts sustained by information gathered in the first observations and bibliographical studies. In this step, we connected our primary observations with scholarship in the main areas of this project, some of which will be presented in the next sections. By doing so, we obtained a better overview of the practices and possibilities of study and were able to define the required characteristics for our sample.

Our third step was the selection of communities and participants, and obtaining informed consent. Based on our initial concepts, we could select the relevant communities and participants, guided by the six requisites proposed by Kozinets (2010): they should be relevant, active, interactive, substantial, heterogeneous and data-rich. Following these guidelines we selected three Facebook groups: Sou autista... conheça meu mundo, Grupo Asperger Brasil, and Lagarta vira pupa (free translation: I am autistic... know my world, Group Asperger Brazil, and Caterpillar turns into pupa). After selecting the groups, we asked for permission to the moderators, but as all the groups had a great number of active members, it was impossible to collect consent from all individual members. Therefore, we announced the research on the Facebook pages, which was warmly received by the community members. However, as in an online setting it is impossible to be sure that all members are fully aware that they are part of a study, we never directly quote from the communities nor present information that can be traced back to individuals, to avoid exposing participants who were not aware of the research.

A fourth step implied entrance into the field and initial participant observation. At this stage, the first author of this paper, who conducted the participant observation and data collection, presented herself to the communities using her personal Facebook profile, as she wanted to be part of the groups and not only exchange information as a researcher. As mentioned before, members of the different groups received the research really well and we experienced no problems to collect the data. After the entrance, the first 
author spent one year in the communities collecting data, notes and quotes from the groups as well as weekly samples, but it was not necessary to collect huge sets of data as all the information was available in an online archive and could be easily found through search engines. After the year of data collection, she did not leave the communities but transitioned from a researcher to a supporter of the autism community.

\section{Autism and Autistic Communities in Brazil}

On the Internet, we can distinguish two kinds of communities in relation to autism: autistic communities, formed by autistic people themselves, and autism communities, which beside autistic people also comprise 'neurotypicals' (i.e. people with a mainstream brain). Usually autistic communities are not exclusory, but they make it clear that their group is made by and for autistic people. In these communities, neurotypical instances that may be common in the autism community are subject to criticism, for instance topics about cures or treating autism as a pathology.

During the initial mapping of this research, we used a wide approach to social media, leading to the observation of multiple platforms before deciding which one(s) best fit the aims of a digital ethnography by being data rich and relevant for the group studied. We could observe Brazilian autism communities across different platforms, but most tend to converge on Facebook, where people manage to meet despite geographical distances and frequently form Whatsapp groups. For instance, it is common to have a Facebook page for a blog or organisation and also have videos published on YouTube but promoted through Facebook. The majority of blogs (including videoblogs and written blogs) that we found were maintained by parents of autistic people, with only a small number of blogs authored by autistic people. One key example is Lagarta vira pupa, written by Andrea Bonoli, the mother of an autistic boy who became an influent person in the autism community because of her blog, which lead to the publication of a homonymous book. Her blog is associated to a Facebook group and her discourse is able to change the views of other parents, as we will discuss later.

The fact that online connections converge around Facebook is not a particularity of the autism community, as this is the most popular platform in Brazil. In a digital ethnographic study, Syper (2013) mentioned that in Brazil "the Internet is Facebook", as people usually open their mobiles to access this particular social media. The use of Whatsapp is also strong and Brazilians tend to spend a lot of time using social media and texting.

Despite being a mostly text-based form of communication, Facebook has affordances that allow communication without requiring a high level of digital literacy or even literacy. People can post short written texts or videos and pictures, and they can interact through reactive buttons only, such as liking and sharing. Moreover, Facebook allows the integration of Instagram, Twitter, and YouTube, the other three popular social media platforms in the country, which makes cross-posting a frequent practice.

After analysing the platforms and deciding to focus on Facebook communities, we observed the groups that operate there. We firstly found that the number of groups of parents (autism communities) was greater than the ones formed by autistic people themselves (autistic communities), but we were glad to see that during our research, these numbers were increasing, with some groups being restricted to autistic 
people. Although there were quite a few groups of parents, most of them tended to have the same active participants, with the exception of groups that were therapy specific, such as the ones focusing on a gluten and casein free diet. We observed that Sou autista... conheça meu mundo (SACM) was one of the most participative, containing a wide range of discourses and also including autistic people. We also wanted to see how the tone of a page or initial post would shape the conversations, so we chose the group Lagarta vira pupa, which offers a more positive perspective on autism. We also selected the Grupo Asperger Brasil for being one with a high number of autistic people without being closed and reserved only for autistic people.

After becoming part of the groups and during the year of participant observation, we found two issues that are worth discussing: the main intentions of people when they join autism communities and what they can find there; and the themes discussed in the communities and how autism was framed in these discussions.

First, the main reasons why parents and autistic people look for communities about autism on Facebook is to find information and social support, which is in line with other studies about disability and social media (Clifford, 2011). The groups provide them with information without the mediation of professionals, so what is shared is mainly based on experience. In the groups we observed, many parents complain about the lack of professionals that understand autism, hence the community is a chance to find information that they may not find where they live - given the fact that Brazil is a huge country, and inequality as well as access to health care varies across region and between the capitals and the countryside. For instance, most of the best institutes are situated in two capitals of the South-East, Rio de Janeiro and São Paulo. Although there is a network of treatment for psychosocial problems in Brazil, CAPS (Centro de Atenção Psicossocial, free translation: Centre of Psycho-social Attention), many claim that the professionals are not instructed to deal with autism. Moreover, both parents and autistic people look for people like themselves, which whom they can share their experiences and learn from others'.

Apart from information, parents also look for social support; in the groups they can find an environment where they can feel included, which may be denied to them in the offline world. Many parents report in the communities that even their own families started to exclude them after their son or daughter was diagnosed with autism, some family members arguing that the kid has nothing and is just spoiled, others preferring not to get close nor offer any help when parents are in need. For instance, a few mothers complained that while other kids in the family were taken to social gatherings, the autistic ones were never invited.

Second, concerning the themes discussed in the communities and the way autism was framed in them, we observed that the groups share some themes but we want to present each one individually to do justice to their specificity. In SACM most of the posts relate to the situations parents, mainly mothers, go through with their autistic children. There are a great number of posts talking about daily life challenges, such as inclusion in school, prejudice among other people and even the extended family, therapies and medicines, and difficulties in getting treatment. As an example, one mother once posted:

I took my son to play in a playground, but unfortunately the prejudice against kids with special needs is everywhere, coming from both adults and other kids. One kid said to his mother that he was afraid of my son and the mother replied that she was 
going to protect him, as if my kid was some sort of threat (free translation extracted from SACM).

Those posts are usually received with empathy by other members, who often share the same challenges, and there is an exchange of social support in the comments. In this particular example, many mothers suggested how she could have reacted or related that they experienced similar situations. Parents there commonly refer to their kids as angels, as we can see in one reply to the post above: "I have experienced the same situation with my angel, but I no longer have patience, I am fighting for my son". Although parents use the term angel to demonstrate love and caring, some autistic people argue that it contributes to the infantilisation of disabled people.

Parents on SACM are also eager to learn about the medicines other children are taking and the reactions to and advantages of medicines prescribed to their own children. Pictures circulate freely in the community, with parents posting photos of their daily lives with their children or just of their children (e.g. when they go to school for the first time, when they are eating by themselves, and birthday pictures). Those images are quite important as Brazil still suffers from a lack of digital literacy, and even general literacy, and pictures offer an easy way to communicate and express emotions without the need of literacy (Miller et al., 2016). Moreover, pictures have the power to work as emotional carriers (Barthes, 1981), by sharing intimate moments in a straightforward manner, which may induce people to feel more connected to each other.

Overall, the tone of the discussions in SACM is more connected to the view of autism as a pathology, with only a few parents trying to introduce the idea of neurodiversity, which considers autism as part of human's neurological differences rather than as a pathology (Jaarsma \& Welin, 2012). As such, the community can be characterised as strongly oriented towards social support. The narratives in this group are dealing with private aspects of parents' lives, usually filled with emotions: sometimes frustration with a society that excludes their children or with the daily challenges they face, at other times happiness with the accomplishment of achievements. By spending a large amount of time in this community, we soon got in touch with the private lives of many parents who are more active participants. We also learned about the stories and perspectives shared which enabled us to contribute to the discussions.

Like SACM, Lagarta vira pupa is also mostly composed of parents of autistic children. However, Andrea Bonoli, the administrator and owner of the weblog to which the group is associated, has a positive perspective of autism more associated with the idea of neurodiversity. The discussions respond to her initial posts, which talk about her daily life with Theo, her autistic son. In one post she refers back to an article she wrote (Bonoli, 2014) about getting the autism diagnosis and how it was difficult, but that she wishes that other people's mourning over the diagnosis would not last long and that they could find their way through it. Although the text talks about all the challenges of dealing with the diagnosis for the first time, it ends with a bright note about progress and never underestimating an autistic person. Many mothers replied to this message saying how the text softened their pain and how it helped them to deal with the autism diagnosis. Obviously not all the messages are promoting acceptance, one mother replies "I did not expect one of my biggest fears to become reality (referring to autism). But, as all in my life, I could not wait for the good. The worst case always happen and gets me along the way", but the majority are thanking Andrea for her words and showing that they believe their kids can have a good life. 
Andrea's experiences do not deny the challenges of autism, but she deals with them in a positive way and also encourages and supports other parents. For instance, she writes posts about therapies, lists of inclusive schools, and doctors - some in a collaborative manner with her readers. As in SACM the discussions are based on the participants' similar private lives of having a family member with autism. However, the two groups have an interesting difference in terms of how the initial tone of the conversation invites a particular kind of reply. While in SACM the pain of many parents generates more pain and pity, Andrea's positive view impacts the parents' reactions as they show themselves to be more willing to face their difficulties by looking at the bright side or focusing on the outcomes instead of the drawbacks. Although parents in SACM also share their happiness, the general environment is more positive in Lagarta vira pupa. Andrea welcomes the members of the group in her private life, as the Facebook page is permeated with pictures of her family and daily life, while members also welcome Andrea to their lives by sharing their own perspectives and routines.

Meanwhile, Grupo Asperger Brasil presents a different kind of interaction than the other two communities, as it is mostly (but not only) constituted of autistic people talking about their lives, so it leans more towards an 'autistic' than an 'autism' community. The narratives tend to be more about how autism impacts their lives in terms of personality traits. Most of the posts ask other people if they have similar characteristics and how they cope with them. There are also a number of posts addressing the challenges in social interaction they face; members often narrate how it is difficult to make new friends, to adapt in school, and to find romantic relationships. One member once asked:

Hello fellows. Today I would like to know what are the biggest challenges when you try to maintain or initiate a relationship. I admit that, in my case, the main problems are to meet my partner's family or interacting in my partner's social circle, for people who had only tried with non-aspies, as me, the attempts are often stopped at these stages. I also find it difficult to interact with my own extended family. But this is a theme for another post. Have a good day.

A few people replied to the post reporting that they also have problems to interact even with the person that they are interested in and they also find it challenging to express their emotions in a way that other people can comprehend and do not find them weird or rude. Most people who replied affirm that they feel inferior because of these problems and think they have to live alone. In this case, they provided support and affirmed that none of them were inferior, however there was not much advice to be given, but more an exchange of personal experiences. One interesting note is that topics reporting their experiences tend to be long and more elaborated in this group than in the others, showing a characteristic of good written articulation by autistic people and preference for details.

There are also discussions about the mental comorbidities associated with autism, such as depression and anxiety. Compared to the other communities, and despite the focus on the challenges caused by autism, in this group there are a high number of members who adopt the neurodiversity perspective, considering autism as a neurological difference rather than a disease. 


\section{Discussion and conclusion}

The autism community on Facebook in Brazil presents a great number of parents, against a small number of autistic people, who are often Aspergers or high-functioning autistic people. For instance, we did not find any non-verbal autistic during our research. Those people, autistic people and parents, are using social media in the same way as many minority groups, to find social support, gather information, exchange experiences, and find similar others. However, the inclusion that social media brought to autistic people can make a great difference in their lives, as they are able to communicate in a manner that is more suitable for them. Thus, although we can argue that they use social media in similar ways as neurotypicals, social media do add another layer to their lives that cannot be compared to the neurotypical usage, as it mediates their challenges in communication.

In the case of the communities of parents, we also noticed a discrepancy in the presence of mothers and fathers: although there are a few active fathers, most of the communication is carried out by mothers, who are usually responsible to provide the support to their daughters and sons. The most common themes in the communities with a predominant presence of parents are related to medical perspectives, as they are mostly looking for advice or information about therapies and medicines or similarities in behaviours with other autistic people. There are also many posts about the social exclusion they and their daughters and sons face because of autism, such as prejudice in the school environment. A great number of mothers report feelings of fatigue and depression because of their hectic daily lives, which is in line with research that found that mothers of autistic kids face a high level of stress in Brazil (Gomes et al., 2015). Moreover, posts about the socio-economic benefits that the government should allocate to autistic people are common, as many families face financial difficulties and struggle to find appropriate support.

Meanwhile, the community we studied with a greater number of autistic people often has posts looking for similarities and the exchange of personal experiences. Unfortunately, a high number of the active members are struggling with mental issues, so posts regarding that matter are also common. Other autistic people can offer personal advice and tell how they dealt with specific problems in their lives. It is also motivating to see that many succeeded in their careers and personal lives, taking advantage of the benefits of the autistic mind and finding ways to cope with its challenges. During the time we collected data, members have been changing frequently, as it was an open group and some autistic people argued that they felt uncomfortable with the high presence of neurotypicals - mainly parents of autistic people. Thus, we noticed other more restricted groups being formed, but we did not want to collect data in those more private venues out of respect for their privacy.

Across the autism community, we could see an embryonic movement towards advocacy, usually through posts of autistic people or parents who are engaged with activism, such as Cristiano Camargo (2012), an autistic writer who promotes neurodiversity in many communities and is a well-known figure in the scene, as well as the mothers Deusina Lopes (2008) and Andrea Bonoli (2016), both of whom published books about autism, and Manuel Vasquez Gil, also a father of an autistic boy and psychanalyst who has published about autism and works with inclusion. Although we talk about the empowering potential of social media, this movement is slowly spreading, as autism is still mostly seen from the medical and pathological perspective. We noticed that an important fact for the promotion of neurodiversity is the coexistence of autistic adults and parents in the same online environment, so one can group can help the 
other to better understand the peculiarities of autism. Obviously this coexistence does not come without conflicts, as many autistic people have already reported feelings of anger and annoyance at parents who complain about autism and treat it as evil, since autistic people say that they cannot detach autism from their personalities and when parents say that they hate autism, they are implying that they also hate autistic people. From their side, parents are keen to defend themselves by saying that they love their children, but not all the limitations of autism. There is no right view on these matters, but, despite the conflicts, it is important to have the different opinions open for everybody.

We find it important to emphasise the exclusion of people along the spectrum. During our research we had contact with the autism community of other countries, such as the United Kingdom, Canada and the United States, where policies for the inclusion of people with disabilities are more advanced; and in such places we could find a number of autistic individuals who are not just at the high end of the spectrum. Thus we understand that better policies are required to increase access to the autistic community, both in terms of digital literacy, and even literacy - as the education system is still problematic when it comes to inclusion, and socio-economical support.

With this paper we hope to have given an overview of the digital scene of autism in Brazil. In further works we are going to explore the question of identities and digital culture, as well as the tools provided by social media to autistic people. We want to finish by acknowledging that we understand that cyberspace is broader than Facebook and also that an offline ethnography could greatly contribute to the comprehension of autism in Brazil. However, we do expect that the field of critical autism studies and disability studies in general can grow in Brazil and in the Global South, helping us to better understand and include diversity in the agenda of those places, also addressing the difficulties faced by disabled people and the benefits that their views can bring to society.

\section{References}

Barthes, R. (1981). Camera Lucida: Reflections on Photography. New York: Hill and Wang.

Beneito-Montagut, R. (2011). Ethnography Goes Online: Towards a User-Centred Methodology to Research Interpersonal Communication on the Internet. Qualitative Research, 11(6), 716-735.

Benford, P, \& Standen, P. (2009). The internet: a comfortable communication medium for people with Asperger syndrome (AS) and high functioning autism (HFA)? Journal of Assistive Technologies, $3(2), 44-53$.

Boellstorff, T. (Org.). (2012). Ethnography and Virtual Worlds: A Handbook of Method. Princeton: Princeton University Press.

Bonoli, A. (2016). Lagarta Vira Pupa. Santa Catarina: CR8 Editora.

Bonoli, A (2014) Meu desejo. Retrieved 14 March 2018, from www.lagartavirapupa.com.br/meu-desejo/

boyd, D. (2011). Social Network Sites as Networked Publics: Affordances, Dynamics, and Implications. In Paparacharissi, Z. (ed). A Networked Self: Identity, Community, and Culture on Social Network Sites (pp. 39-58). New York: Routledge.

Bowker, N. (2003). What it Means to be Online for People with Disabilities (Doctor of Philosophy in Psychology). Massey University, Palmerston North, New Zeland. 
Brownlow, C., \& O'Dell, L. (2006). Constructing an Autistic Identity: AS Voices Online. Mental Retardation, $44(5), 315-321$.

Camargo, C. (2012). Autista com Muito Orgulho. FDigital.

Carter, I. (2009). Positive and Negative Experiences of Parents Involved in Online Self-Help Groups for Autism. Journal on Developmental Disabilities, 15(1), 44-52.

Clifford, T. (2011). Support Groups for Parents of Children with Autism Spectrum Disorders: Predictors and Effects of Involvement (Doctor of Philosophy in Psychology). Queen's University, Kingston, ON.

Davidson, J. (2008). Autistic Culture Online: Virtual Communication and Cultural Expression on the Spectrum. Social \& Cultural Geography, 9(7), 791-806.

Davidson, J., Edwards, S., \& Hemsworth, K. (2012). Autism Online - The Role of the Internet in the Daily Lives of People on the Autism Spectrum (p. 28). Kingston, ON: Queen's University.

Dekker, M. (2006, novembro 11). On Our Own Terms: Emerging Autistic Culture. Recuperado 23 de fevereiro de 2016, de http://web.archive.org/web/20061111053135/http://trainland.tripod.com/martijn.htm

Ellis, K., \& Goggin, G. (2015). Disability and the Media. New York, NY: Palgrave Macmillan.

Gomes, P. T. M., Lima, L. H. L., Bueno, M. K. G., Araújo, L. A., Souza, N. M., Gomes, P. T. M., ... Souza, N. M. (2015). Autism in Brazil: a systematic review of family challenges and coping strategies. Jornal de Pediatria, 91(2), 111-121.

Jaarsma, P., \& Welin, S. (2012). Autism as a Natural Human Variation: Reflections on the Claims of the Neurodiversity Movement. Health Care Analysis, 20(1), 20-30.

Hine, C. (2000). Virtual Ethnography. London; Thousand Oaks, Calif: SAGE.

Kozinets, R. V. (2010). Netnography: Ethnographic Research in the Age of the Internet (1st ed). Thousand Oaks, CA: Sage Publications Ltd.

Lister, M. et al. (2009). New Media: A Critical Introduction (2nd ed). Milton Park, Abingdon, Oxon; New York, N.Y: Routledge.

Lopes da Cruz, D. (2008) Um Autista Muito Especial. Porto Alegre: Mediação.

Meekosha, H. (2011). Decolonising Disability: Thinking and Acting Globally. Disability \& Society, 26(6), $667-682$.

Miller, D. et al. (2016). How the World Changed Social Media. London: UCL Press.

Newton, A. T., Kramer, A. D. I., \& McIntosh, D. N. (2009). Autism Online: A Comparison of Word Usage in Bloggers with and Without Autism Spectrum Disorders. In Proceedings of the SIGCHI Conference on Human Factors in Computing Systems (p. 463-466). New York, NY, USA: ACM.

Ortega, F., Zorzanelli, R., Meierhoffer, L. K., Rosário, C. A., Almeida, C. F. de, Andrada, B. F. da C. C. de, ... Feldman, C. (2013). A Construção Do Diagnóstico Do Autismo Em Uma Rede Social Virtual Brasileira. Interface - Comunicação, Saúde, Educação, 1744), 119-132.

Recuero, R. (2011). Redes Sociais Na Internet. Porto Alegre: Sulina.

Reinke, J. S., \& Solheim, C. A. (2014). Online Social Support Experiences of Mothers of Children with Autism Spectrum Disorder. Journal of Child and Family Studies, 24(8), 2364-2373.

Seymour, W., \& Lupton, D. (2004). Holding the Line Online: Exploring Wired Relationships for People with Disabilities. Disability \& Society, 19(4), 291-305. 
Shane-Simpson, C., Brooks, P. J., Obeid, R., Denton, E., \& Gillespie-Lynch, K. (2016). Associations Between Compulsive Internet Use and the Autism Spectrum. Research in Autism Spectrum Disorders, 23, 152-165.

Shpigelman, C.-N., \& Gill, C. J. (2014). Facebook Use by Persons with Disabilities. Journal of ComputerMediated Communication, 19(3), 610-624.

Silberman, S. (2015). Neurotribes: The Legacy of Autism and the Future of Neurodiversity. New York: Avery, a member of Penguin Group USA.

Spyer, J. A. (2013, agosto 9). Is it bad that facebook became the king of communication among Brazil's "new middle class" youth? Retrieved 16 August 2016, from http://blogs.ucl.ac.uk/global-socialmedia/2013/08/09/is-it-bad-that-facebook-became-the-king-of-communication-among-brazilsnew-middle-class-youth/

Stein-Sparvieri, E. (2012). Creencias Y Actitudes En Torno Al Problema De La Discapacidad En Distintos Países De América Y Europa. Subjetividad y procesos cognitivos, 16(1), 218-244.

Sutcliffe, A. G., Gonzalez, V., Binder, J., \& Nevarez, G. (2011). Social Mediating Technologies: Social Affordances and Functionalities. International Journal of Human-Computer Interaction, 2オ11), 1037-1065.

van der Aa, C., Pollmann, M. M. H., Plaat, A., \& van der Gaag, R. J. (2016). Computer-mediated communication in adults with high-functioning autism spectrum disorders and controls. Research in Autism Spectrum Disorders, 23, 15-27.

Vasilopoulou, E., \& Nisbet, J. (2016). The Quality of Life of Parents of Children with Autism Spectrum Disorder: A Systematic Review. Research in Autism Spectrum Disorders, 23, 36-49. 